\title{
Bone mineral density in patients with inherited bone marrow failure syndromes
}

\author{
Roopa Kanakatti Shankar ${ }^{1}$, Neelam Giri ${ }^{2}$, Maya B. Lodish ${ }^{1}$, Ninet Sinaii ${ }^{3}$, James C. Reynolds ${ }^{4}$, Sharon A. Savage ${ }^{2}$, \\ Constantine A. Stratakis ${ }^{1}$ and Blanche P. Alter ${ }^{2}$
}

BACKGROUND: Patients with inherited bone marrow failure syndromes (IBMFS) may have several risk factors for low bone mineral density (BMD). We aimed to evaluate the prevalence of low BMD in IBMFS and determine the associated risk factors.

METHODS: Patients with IBMFS with at least one dual-energy $X$-ray absorptiometry (DXA) scan were evaluated. Diagnosis of each IBMFS, Fanconi anemia (FA), dyskeratosis congenita, Diamond-Blackfan anemia, and Shwachman-Diamond syndrome was confirmed by syndrome-specific tests. Data were gathered on age, height, and clinical history. DXA scans were completed at the lumbar spine, femoral neck, and forearm. BMD was adjusted for height $(H A Z)$ in children (age $\leq 20$ years). Low BMD was defined as a BMD Z-score and $\mathrm{HAZ} \leq-2$ in adults and children, respectively, in addition to patients currently on bisphosphonate therapy.

RESULTS: Nine of thirty-five adults (26\%) and eleven of forty children (27\%) had low BMD. Adults with FA had significantly lower BMD Z-scores than those with other diagnoses; however, HAZ did not vary significantly in children by diagnosis. Risk factors included hypogonadism, iron overload, and glucocorticoid use.

CONCLUSIONS: Adults and children with IBMFS have high prevalence of low BMD. Prompt recognition of risk factors and management are essential to optimize bone health.

nherited bone marrow failure syndromes (IBMFS) are a group of rare genetically heterogeneous disorders associated with varying degrees of bone marrow failure (BMF) and a high risk of cancers including leukemia, myelodysplastic syndrome, and solid tumors (1). Fanconi anemia (FA), Diamond-Blackfan anemia (DBA), dyskeratosis congenita (DC), and Shwachman-Diamond syndrome (SDS) comprise the four major IBMFS. Patients with IBMFS may have several risk factors for low bone mineral density (BMD), including chronic glucocorticoid therapy (2) and iron overload from frequent red blood cell transfusions (3). Endocrine dysfunction with hypogonadotropic hypogonadism, and growth hormone secretory abnormalities as described in FA, may also contribute to low BMD (4). In addition, hematopoietic stem cell transplantation (HSCT) has been shown to be associated with decreased BMD (5).

$\mathrm{BMD}$ is usually reported as $T$-score (compared with the standard reference for Caucasian females, aged 20-29 years), but, in premenopausal women and men younger than 50 years, $Z$-scores are preferred; low BMD is defined as a $Z$-score of -2 or lower (6). Skeletal sites typically included for determination of BMD using dual-energy X-ray absorptiometry (DXA) scan are postero-anterior lumbar spine (L1-L4), femoral neck or total proximal femur and, in some cases, onethird of the radius of the non-dominant forearm (6). Low BMD in children is defined as age-, gender-, and heightspecific BMD $Z$-scores (using a pediatric reference) of $\leq-2$ at the spine or at the proximal femur (7). As BMD is only an areal measure, previous studies in adults have looked at bone mineral apparent density $(\mathrm{BMaD})$ as an alternative volumetric measure $(8,9)$; however, this method is not widely used.

BMD has been previously reported in small groups of patients with IBMFS. Twelve of thirteen adults with FA had low BMD (4). Another study noted normal BMD in children with FA after correction for height age (the age that corresponds to the child's height when plotted at the $50 \%$ ile on the growth chart) (10). Nine of the eleven patients with SDS had osteoporosis (11). Premature osteoporosis was also described in patients with DC (12). To our knowledge, there are no studies of BMD in DBA. We now report the prevalence of low BMD in children and adults with the four major IBMFS, and discuss the associated risk factors.

\section{METHODS \\ Protocol}

The participants were patients with DBA, DC, FA, or SDS enrolled in the National Cancer Institute's (NCI) IBMFS protocol 02-C-0052 (http://www.marrowfailure.cancer.gov); clinicaltrials.gov NCT000 27274). The study was approved by the National Cancer Institute's Institutional Review Board. Written informed consent was obtained in accordance with the Health and Human Services regulation 45 CFR 46. Diagnosis of each IBMFS was based on standard criteria (1), and was confirmed by syndrome-specific tests as described

\footnotetext{
${ }^{1}$ Division of Intramural Research, Eunice Kennedy Shriver National Institute of Child Health and Human Development, NIH, Bethesda, MD, USA; ${ }^{2}$ Clinical Genetics Branch, Division of Cancer Epidemiology and Genetics, National Cancer Institute, NIH, Rockville, MD, USA; ${ }^{3}$ Biostatistics and Clinical Epidemiology Service, Clinical Center, NIH, Bethesda, MD, USA; ${ }^{4}$ Radiology and Imaging Sciences, Clinical Center, NIH, Bethesda, MD, USA. Correspondence: Neelam Giri (girin@mail.nih.gov)

Received 13 January 2017; accepted 15 April 2017; advance online publication 31 May 2017. doi:10.1038/pr.2017.117
} 
previously (13). The diagnosis of DC was not confirmed in three patients with aplastic anemia and very short telomeres from two families in whom the mutated gene was not identified; these patients were designated "DC-like" but were classified under DC for this analysis.

\section{Subjects}

This report includes 75 patients who had at least one DXA scan completed at the Clinical Center of the National Institutes of Health as part of their outpatient evaluation on the National Cancer Institute's IBMFS protocol. There were 40 children (defined as $\leq 20$ years of age) and 35 adults. Data were gathered on age, treatment history, including age at the start of therapy, and duration of therapy with specific reference to glucocorticoid, testosterone, other androgen, estrogen, and bisphosphonate use, and prior HSCT. Data on fracture history, iron overload, and endocrine features (clinical and laboratory) suggestive of hypogonadism were evaluated when available. The diagnosis of hypogonadism in post-pubertal children and adults was confirmed on the basis of the endocrinology consult note or evidence of clinical and laboratory findings as previously described (4). Iron overload was diagnosed by high serum ferritin and confirmed using $\mathrm{T} 2^{\star}$-magnetic resonance imaging of the liver, SQUID (superconducting quantum interference device), or liver biopsy.

\section{Measurements}

Weight was obtained using a digital scale (Life Measurements Instruments, Concord, CA) and height (measured in triplicate to the nearest $\mathrm{mm}$ ) was obtained using a Harpenden stadiometer (Holtain Ltd., Crymych, UK) calibrated before each set of measurements. Height was expressed as a $Z$-score specific for age and gender, based on National Center for Health Statistics data (14). The measurements were recorded on the same day as the DXA scan.

BMD was measured by DXA scan (QDR-4500A; Hologic, Bedford, $\mathrm{MA}$ ) at the postero-anterior lumbar spine (L1-L4; referred to as "spine BMD"), femoral neck, total hip, and forearm (1/3 radius). In subjects with multiple BMD measures, the first available measure was included for this study. Age- and gender-specific height-adjusted BMD Z-scores (HAZ) were calculated in children using the online Bone Mineral Density Childhood Study calculator (15). We used the age cutoff of 20 years to define children in this study as peak bone mass is achieved after the age of 20 years (the adult $T$-score reference standard is a white female of 20-29 years (ref. 6)) and the Bone Mineral Density Childhood Study calculator provides HAZ estimates up to the age of 20 years (ref. 15). In adults, standard BMD $Z$-scores adjusted for gender, age, and ethnicity were calculated. $\mathrm{BMaD}$ scores were computed in adults only, as a volumetric measure of bone density using the formula: Spine $\mathrm{BMaD}=\mathrm{BMC} /(\text { area })^{3 / 2}$ and femoral neck/forearm $\mathrm{BMaD}=\mathrm{BMC} /(\text { area })^{2}($ refs 8,9$)$.

Low BMD in this study was defined as one of the following: HAZ at the spine or femoral neck $\leq-2$ in children, and spine or femoral neck BMD $Z$-score $\leq-2$ in adults, or if patients were already on bisphosphonate therapy for low BMD at the time of DXA scan.

\section{Data Analysis}

Data were described by frequency distributions and percentages, and simple descriptive statistics where results are reported as mean $\pm \mathrm{SD}$, or median (interquartile (25th, 75th percentile) range), and as relevant for informative purposes, range (minimum to maximum). Categorical data were compared using Fisher's exact test. Comparisons between (i.e., hypogonadism) or among (i.e., syndrome) groups were carried out by $t$-test or analysis of covariance, respectively, with post hoc pairwise comparisons corrected with the Bonferroni method. Correlation analyses involved Pearson's correlation coefficient. Univariable (simple) and multivariable (multiple) regression models assessed the predictive relation between relevant covariates and bone parameter outcomes. All data were assessed for their distributional assumptions, and all tests were two-sided with statistical significance set at $P<0.05$. Where applicable, $P$ values corrected for multiple comparisons are reported. Data were analyzed using SAS v9.2 (SAS Institute, Cary, NC).

\section{RESULTS}

\section{Patient Characteristics}

Out of 75 patients, 15 had DBA, 30 had DC, 24 had FA, and 6 had SDS (Table 1). Thirty-four (45\%) patients were female. Forty were children (median age: 13.0 years; range: $7.6-19.8$ ). The median age of adults was 30.4 years (range: 20.6-69.0). Patients with FA and SDS were shorter than patients with DC $(P<0.05$; Table 1$)$, whereas those with DBA were similar to

Table 1. Demographic and bone mineral density characteristics in patients with IBMFS

\begin{tabular}{|c|c|c|c|c|c|}
\hline Parameter & DBA & DC & FA & SDS & All IBMFS \\
\hline$N(\%)$ & $15(20 \%)$ & $30(40 \%)$ & $24(32 \%)$ & $6(8 \%)$ & 75 \\
\hline Male:female & $10: 5$ & $20: 10$ & $8: 16$ & $3: 3$ & $41: 34$ \\
\hline Range (years) & 8.5-58.4 & $7.6-69$ & $8.1-56.6$ & 8.2-30.4 & \\
\hline \multirow[t]{2}{*}{ Height $Z$-score ${ }^{\mathrm{a}}$ mean $( \pm \mathrm{SD})$} & $-0.94(1.25)$ & $-0.06(1.12)$ & $-1.62(1.35)$ & $-2.24(2.18)$ & $-0.91(1.51)$ \\
\hline & Adults/children & Adults/children & Adults/children & Adults/children & Adults/children \\
\hline Hypogonadism & $3 / 0$ & $0 / 1$ & $11 / 2$ & 0 & $14 / 3$ \\
\hline HSCT & 0 & $0 / 2$ & $3 / 4$ & 0 & $3 / 6$ \\
\hline Oral glucocorticoids & $0 / 5$ & $0 / 2$ & $1 / 0$ & 0 & $1 / 7$ \\
\hline Inhaled steroids & 0 & $0 / 3$ & $2 / 1$ & $0 / 2$ & $2 / 6$ \\
\hline
\end{tabular}

BMD, bone mineral density; DBA, Diamond-Blackfan anemia; DC, dyskeratosis congenita; FA, Fanconi anemia; HSCT, hematopoietic stem cell transplant; IBMFS, inherited bone marrow failure syndrome; IQR, interquartile range (25th percentile, 75th percentile); SDS, Shwachman-Diamond syndrome.

${ }^{a} P<0.001$ for overall height $Z$-score differences, and $P<0.05$ for each of SDS and FA vs. DC (post hoc comparison). 


\section{Articles | shankar et al.}

those with DC. Hypogonadism was present in 14/35 (40\%) adults ( $3 \mathrm{DBA}$ and $11 \mathrm{FA}$ ) and in $3 / 40$ children (7.5\%; $1 \mathrm{DC}$ and 2 FA). Six children (two DC, four FA) and three adults (FA) had undergone HSCT. Eight of seventy-five patients were on oral glucocorticoid therapy (five DBA, two D, one FA) and eight were on inhaled glucocorticoids (three DC, three FA, and two SDS). Nine (six DC and three FA) patients (three males and six females) were on androgen treatment (details in Supplementary Tables S1 and S2 online). Five adult females (two DC and three FA) and four girls with DC were on estrogen/progestin therapy for ovarian insufficiency and menorrhagia, respectively.

\section{BMD Distribution}

The overall prevalence of low BMD was 27\% (20/75). There was no significant difference in the prevalence of low BMD by IBMFS diagnosis $(P=0.8)$, or when stratified by children $(P=0.9)$ and adults $(P=0.9$; Table 1$)$.

Eleven of the forty children (28\%) had low BMD (Supplementary Table S1). One child with DC had both spine and femoral neck HAZ $\leq-2$, nine children (one DBA, five DC, two FA, and one SDS) had a femoral neck HAZ $\leq-2$ but spine BMD HAZ $>-2$, and one child with FA had a history of vertebral compression fractures and low BMD 2 years prior to the study; his spine and femoral neck HAZ scores were normal on alendronate therapy at the time of this study. Three of the eleven children with low BMD (two DC and one FA) were on chronic oral glucocorticoid therapy (one for pulmonary fibrosis and the other two for BMF), and three had transfusion-associated iron overload (one DBA and two DC), two (with FA) were 4 and 10 years post-HSCT, whereas three (two DC and one SDS) had not been on any treatment.

Prevalence of low BMD in adults was 26\% (9/35; Supplementary Table S2). Spine and femoral neck BMD $Z$-scores were $\leq-2$ in two adults (one DC and one FA), four had only spine BMD $Z$-score $\leq-2$ (one DC and three FA), and one (DBA) had femoral neck BMD $Z$-score of -2 . Seven of the nine adults with low BMD had hypogonadism (five FA and two DBA). Four were on bisphosphonates: one each with DBA and FA had been on alendronate treatment for over 7 years and had normal BMD $Z$-scores at the time of study, whereas two others (both FA) had BMD $Z$-score $\leq-2$ despite undergoing alendronate therapy for 2 years; one of them had also developed vertebral compression fractures. Three of the nine adults with low BMD had iron overload (two DBA and one $\mathrm{DC}$ ), one (FA) had been on prednisone (plus androgen) for over 10 years, and one (DC) was not on any treatment. All five adults with FA with low BMD as well as the two adults with DBA with low BMD had hypogonadism.

Table 2 shows the distribution of BMD in adults and children by diagnosis and site of BMD measurement, and Figure 1 shows the distribution of lumbar spine BMD. There were no statistically significant differences in HAZ at the spine $(P=0.12)$, femoral neck $(P=0.18)$, or forearm

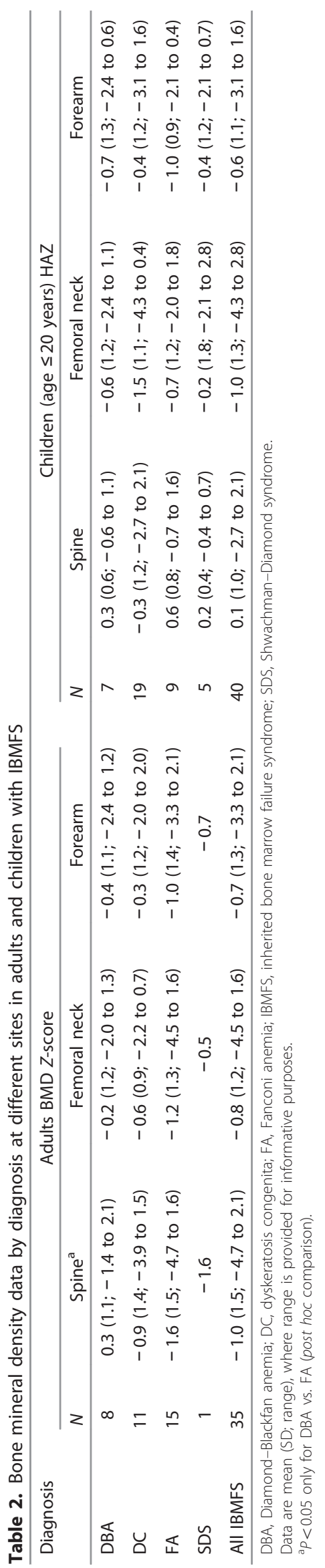




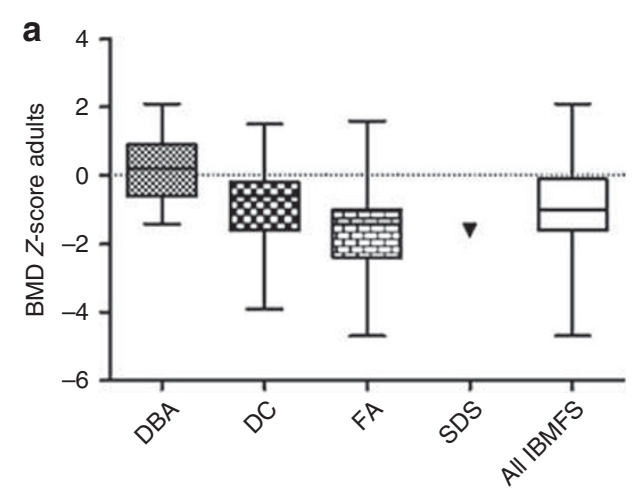

Diagnosis

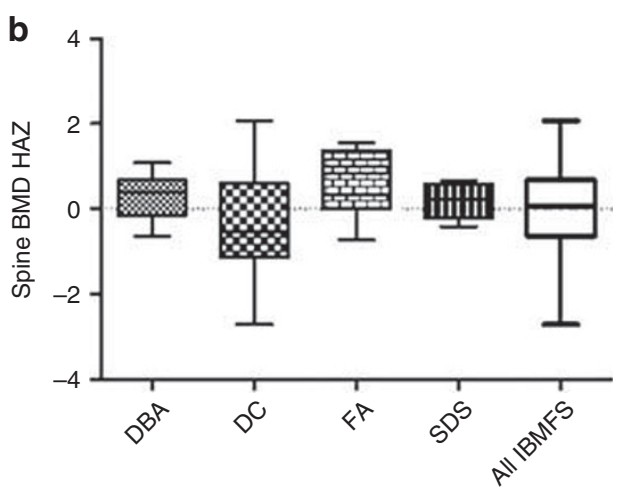

Diagnosis

Figure 1. Distributional plot of BMD at the lumbar spine by diagnosis (a) in adults and (b) in children. In adults, (a) BMD Z-score was statistically significantly different by diagnosis ( $P=0.034$, FA vs. DBA driving the significant difference). The arrowhead reflects the BMD Z-score of the one adult patient with SDS. (b) There were no statistically significant differences in BMD HAZ $(P=0.12)$ among diagnoses in children; data at lumbar spine are shown. BMD, bone mineral density; DBA, Diamond-Blackfan anemia; FA, Fanconi anemia; HAZ, height-adjusted BMD Z-score.
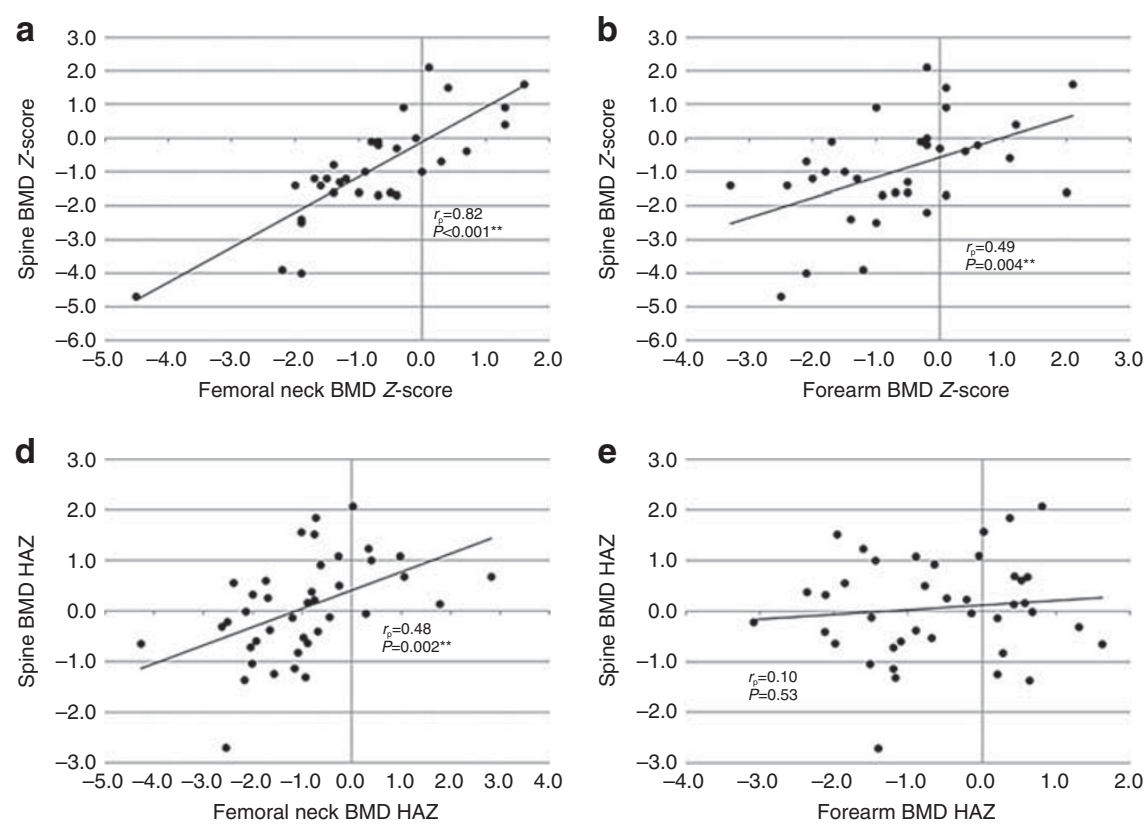

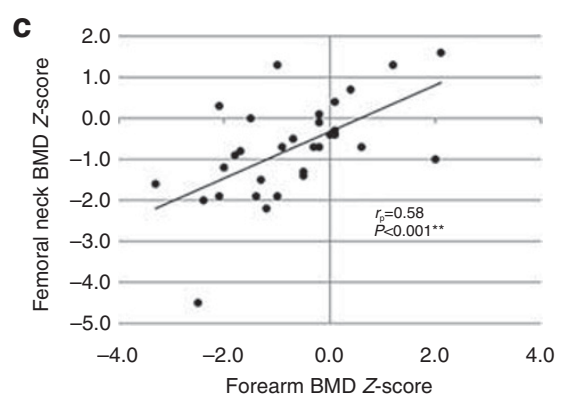

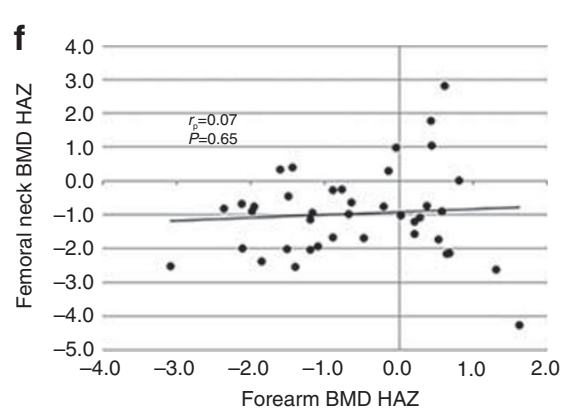

Figure 2. Correlation of BMD measurements at different sites. In adults, (a) femoral neck BMD Z-score vs. spine BMD Z-score; (b) forearm BMD Zscore vs. spine BMD Z-score; (c) forearm BMD Z-score vs. femoral neck BMD Z-score. In children, (d) femoral neck BMD HAZ vs. spine BMD HAZ; (e) forearm BMD HAZ vs. spine BMD HAZ; (f) forearm BMD HAZ vs. femoral neck BMD HAZ. ${ }^{* *} r_{p}$ is the Pearson correlation coefficient. BMD, bone mineral density; DBA, Diamond-Blackfan anemia; FA, Fanconi anemia; HAZ, height-adjusted BMD Z-score.

$(P=0.53)$ in children with the four syndromes. In adults, there were significant differences only in BMD $Z$-score at the spine ( $P=0.034$, FA vs. DBA driving the difference) but not at the femoral neck $(P=0.25)$ or forearm $(P=0.49)$ by diagnosis.

\section{Correlation of BMD and BMaD Measures with Height in Adults} with IBMFS $(\mathrm{N}=35)$

Spine BMD Z-score was strongly correlated with height (Pearson correlation coefficient, $r_{\mathrm{p}}=0.55, P<0.001$ ) but spine $\mathrm{BMaD}$ was not $\left(r_{\mathrm{p}}=-0.01, P=0.95\right)$. Similarly, femoral neck BMD Z-score was strongly correlated with height $\left(r_{\mathrm{p}}=0.48\right.$,
$P=0.005)$ but femoral neck $\mathrm{BMaD}$ was not $\left(r_{\mathrm{p}}=0.13\right.$, $P=0.46$; Supplementary Figure S1).

\section{Correlations of Multiple Site Measures}

BMD measurements from the three different sites were relatively well correlated (Figure 2): spine and femoral neck BMD Z-scores $\left(r_{\mathrm{p}}=0.82, P<0.001\right)$ in adults (Figure 2a) were highly correlated, as were spine and forearm BMD $Z$ scores $\left(r_{\mathrm{p}}=0.49, P=0.004\right.$; Figure $\left.2 \mathbf{b}\right)$ and forearm and femoral neck BMD $Z$-scores $\left(r_{\mathrm{p}}=0.58, P<0.001\right.$; Figure $\left.2 \mathrm{c}\right)$. In children, spine and femoral neck HAZ were correlated $\left(r_{\mathrm{p}}=0.48, P=0.002\right.$; Figure $\left.2 \mathrm{~d}\right)$, but forearm 
BMD HAZ was not correlated with spine $\left(r_{\mathrm{p}}=0.10, P=0.53\right.$; Figure 2e) and femoral neck $\left(r_{\mathrm{p}}=0.07, P=0.65\right.$; Figure 2f) BMD HAZ.

\section{Factors Associated with Low BMD}

HSCT. Three of thirty-five (9\%) adults, all with FA, had undergone HSCT. Average time since HSCT was 11.5 years $( \pm 5.3$, range 5.6-15.9). The mean spine BMD Z-score was -2.4 $( \pm 2.0$, range -4.7 to -1.2$)$ in adults with HSCT compared with -0.8 ( \pm 1.4 , range -4.0 to 2.1$)$ in non-HSCT adult patients; however, this was not statistically significant $(P=0.08)$. However, adults who underwent HSCT had lower femoral neck BMD $Z$-scores (mean $-2.5 \pm 1.7$, range -4.5 to -1.3 vs. mean $-0.6 \pm 1.0$, range -2.2 to $1.6 ; P=0.007)$ than those who did not undergo HSCT. Six of forty (15\%) children underwent HSCT (two DC and four FA); neither mean spine BMD-HAZ nor femoral neck BMD-HAZ was statistically significantly different between the two groups (spine: $0.0 \pm 1.0$, range 2.7 to 2.1 vs. $0.2 \pm 0.8$, range -0.7 to $1.2, P=0.7$; femoral neck: $-1.1 \pm 2.1$, range -4.3 to 1.8 vs. $-0.9 \pm 1.2$, range -2.6 to $2.8, P=0.8$ ).

Hypogonadism. Fourteen of thirty-five adults (40\%) had hypogonadism (Table 1). Spine BMD Z-score (mean $-2.1 \pm 1.3$, range -4.7 to -0.2 ) in those with hypogonadism was significantly lower than in those without hypogonadism (mean $-0.2 \pm 1.1$, range -2.2 to $2.1 ; P=0.001$ ). Femoral neck BMD $Z$-score was also lower in adults with hypogonadism compared with those without hypogonadism (mean $-1.7 \pm 1.0$, range -4.5 to -0.4 vs. mean $-0.1 \pm 0.9$, range -1.3 to 1.5 ; $P<0.001)$. Several children were pre-pubertal, and conclusive determination of hormonal status was only possible in 18/40 (45\%), of whom 3 (17\%) had hypogonadism.

Steroids. The number of patients on glucocorticoids (13 children and 3 adults), androgens (five children and four adults), and estrogen/progesterone (four children and five adults) therapies was relatively small. For adults, a multivariable regression model incorporating the predictors of height, HSCT, steroids, diagnosis, and hypogonadism explained $40 \%$ of the variability in spine BMD $Z$-score $(P=0.001)$. These covariates together explained more of the variability in femoral neck $Z$-score in adults $(65 \%, P<0.001$; Table 3$)$. In children, the small numbers precluded additional analysis.

\section{DISCUSSION}

This study provides the first comprehensive description of bone mineral density in a cohort of patients with IBMFS. Twenty-eight percent of the children have low BMD even after correction of BMD to height-specific $Z$-scores using a pediatric reference calculator. The median age of children with low BMD was 15.8 years (range: $12.7-19.8$ years). There was no difference in the frequency of low BMD by IBMFS diagnosis (DC vs. FA vs. DBA vs. SDS) among children. If we presume femoral neck measurements to be unreliable in children because of significant variability in skeletal development (7), then, using the spine BMD alone, the prevalence of low BMD was $7.5 \%(3 / 40)$. Screening DXA scan is generally not recommended prior to 14 years of age unless compelled by clinical evidence of osteopenia or history of long-term glucocorticoid use. Identification of low BMD in children with IBMFS is important as some may have vertebral compression fractures as evident in our patients. The nine adults with low BMD (26\%) in our study were mostly young patients (median age 29.8 years; Supplementary Table S2) in whom multifactorial etiologies may have contributed to the increased prevalence of low BMD.

The prevalence of low BMD HAZ in children with FA was $33 \%$. This is contrary to the previous report of normal BMD in children with FA after correction for height age (10). However, the children in our study were slightly older (13-18 years) and two of the three with low BMD HAZ had undergone HSCT. Glucocorticoid therapy, hypogonadism, and growth hormone deficiency may be other contributory factors in FA. We previously showed a high prevalence of primary ovarian insufficiency in young adult women with FA (16). In the current study, premature ovarian failure/ hypogonadism appears to be the predominant underlying

Table 3. Results from simple and multiple regression models for outcomes of spine and femoral neck BMD Z-scores in adults with IBMFS

\begin{tabular}{|c|c|c|c|c|c|c|c|c|}
\hline & \multicolumn{4}{|c|}{ Univariable models } & \multicolumn{4}{|c|}{ Multivariable models } \\
\hline & \multicolumn{2}{|c|}{ Spine BMD Z-score } & \multicolumn{2}{|c|}{$\begin{array}{c}\text { Femoral neck BMD } \\
Z \text {-score }\end{array}$} & \multicolumn{2}{|c|}{ Spine BMD Z-score } & \multicolumn{2}{|c|}{$\begin{array}{c}\text { Femoral neck BMD } \\
Z \text {-score }\end{array}$} \\
\hline & $R^{2}$ & $P$ value & $R^{2}$ & $P$ value & $\operatorname{Adj} R^{2}$ & $P$ value & Adj $R^{2}$ & $P$ value \\
\hline All variables ${ }^{a}$ & & & & & $40.5 \%$ & 0.001 & $65.1 \%$ & $<0.001$ \\
\hline Height & $20.2 \%$ & 0.007 & $22.7 \%$ & 0.005 & - & 0.13 & - & 0.015 \\
\hline $\mathrm{HSCT}$ & $8.9 \%$ & 0.081 & $21.1 \%$ & 0.007 & - & 0.043 & - & $<0.001$ \\
\hline Diagnosis (FA vs. non-FA) & $14.1 \%$ & 0.026 & $11.9 \%$ & 0.0496 & - & 0.19 & - & 0.006 \\
\hline Hypogonadism & $36.4 \%$ & $<0.001$ & $42.2 \%$ & $<0.001$ & - & 0.003 & - & $<0.001$ \\
\hline
\end{tabular}

$\mathrm{BMD}$, bone mineral density; FA, Fanconi anemia; HSCT, hematopoietic stem cell transplantation.

$R^{2}$, coefficient of determination; indicates the percentage of the variability of the outcome that is explained by the model. Univariable (simple) and multivariable (multiple) regression models were used for assessing the predictive relation between relevant covariates and bone mineral density outcomes. All data were assessed for their distributional assumptions, and all tests were two-sided with statistical significance set at $P<0.05$. $P$-values corrected for multiple comparisons are reported; significant $P$-values are in bold.

aHeight, HSCT, steroids, diagnosis, hypogonadism. 
cause of low BMD in adult patients with FA. Five of fifteen adults with FA (33\%) had low BMD. They were all females and had primary ovarian insufficiency; only one had undergone HSCT 16 years previously.

Glucocorticoid therapy, iron overload, and hypogonadism were the likely risk factors for low BMD in DBA. The child with DBA with low femoral neck BMD HAZ was on glucocorticoid therapy and had iron overload, whereas the two adults with DBA with low BMD had hypogonadism secondary to iron overload; one was also on glucocorticoid. No risk factor could be identified in the child with SDS who had low femoral neck BMD but normal spine BMD HAZ.

Thirty-two percent of the children with DC had low BMD HAZ. Glucocorticoid use and iron overload were identified as risk factors (Supplementary Table S1). A male child with DC in our study had multiple long bone fractures reported with minimal trauma but had completely normal BMD measurements. Additional BMD measurement in the lateral distal femur may be considered in such patients as conventional DXA scans alone may be insufficient to predict fracture risk (17). One adult patient with DC had received chelation for iron overload and no apparent cause could be identified in the other adult patient with DC and low BMD.

Iron overload may be a predominant factor causing low BMD, especially in DBA and DC. Although the molecular mechanisms of iron overload resulting in osteoporosis are not well understood, it appears to decrease bone formation and affect the microarchitecture by increasing apoptosis of osteoblasts and impeding their recruitment and function (18). HSCT was associated with significantly lower BMD measures in the femoral neck but not in the lumbar spine in our study. Further studies are warranted to determine whether HSCT differentially affects BMD at different sites, as well as to study the change in BMD seen over time after HSCT. Glucocorticoids have long been known to decrease bone formation by reduced osteoblast formation, increased apoptosis of osteoblasts and osteocytes, with reduced bone turnover from decreased osteoclastogenesis (19). Several patients with low BMD in this study had a history of glucocorticoid use, but the small number of total patients precluded further analysis.

We studied the correlation of BMD Z-scores at the spine and femoral neck, and found that the measures were strongly correlated with height in adults. This raises the possibility of whether adult patients with IBMFS with short stature, particularly those with FA or SDS who have a low height $Z$-score, may be incorrectly diagnosed with low BMD. In the multivariate regression analysis, height did not predict BMD $Z$-score at the spine but did predict femoral neck BMD $Z$-scores $(P=0.015)$. Volumetric $\mathrm{BMaD}$ measures have been proposed for adjustment of stature in adults. Indeed, we noted that $\mathrm{BMaD}$ measures did not correlate with height. However, the use of $\mathrm{BMaD}$ measurements may not add to the management of these patients at this time because of insufficient normative data published on $\mathrm{BMaD}$ and the inability to predict fracture risk. The impact of height on
BMD measurements in adults should, however, raise caution in interpretation. Further assessment of BMD Z-scores in larger populations of IBMFS patients and correlation of diagnosis and measures with fracture risk is warranted.

Management of low BMD should begin with recognition and treatment of the risk factors causing secondary osteopenia such as hypogonadism and iron overload. Treatment of osteoporosis should be undertaken in consultation with an endocrinologist. Bisphosphonates are the typical first line of therapy.

The strength of our study is that it provides the first comprehensive assessment and comparison of BMD in children and adults with the four major IBMFS and addresses the associated risk factors for low BMD. The limitations of our study include the lack of data on cumulative glucocorticoid or androgen doses, and small sample size, which may have contributed to the lack of differences in BMD related to steroid therapy and iron overload. We were unable to distinguish patients with adequately corrected hypogonadism or to quantify the duration of untreated hypogonadism that may be specifically causative of low BMD. Despite these limitations, our data contribute to the understanding of $\mathrm{BMD}$ distribution in patients with IBMFS, the correlation of measures at different body sites, as well as the different factors that may be associated with low BMD.

In summary, our study demonstrates a high prevalence of low BMD in patients with IBMFS. Low BMD in adults with FA was mainly associated with hypogonadism. Early detection of hypogonadism and hormonal supplementation may facilitate adequate bone acquisition in these patients. Iron overload and chronic glucocorticoid therapy are other important causes of low BMD in IBMFS, specifically in patients with DC and DBA. We show a high prevalence of low $\mathrm{BMD}$ in children as well. Whereas low BMD at the femoral neck may suggest a difference in skeletal maturity, low BMD at the lumbar spine may result from iron overload and/or chronic glucocorticoid therapy, which can lead to vertebral compression fractures. Prompt recognition and treatment of low BMD and the associated risk factors are important in the clinical management of these individuals. Future studies are needed to explore the fracture risk in patients with IBMFS and low BMD, as well as the contribution of HSCT and glucocorticoid therapy to the risk of low BMD and osteoporosis in these patients.

\section{SUPPLEMENTARY MATERIAL}

Supplementary material is linked to the online version of the paper at http://www.nature.com/pr

\section{ACKNOWLEDGMENTS}

We thank Lisa Leathwood, RN, Maureen Risch, RN, and Ann Carr, MS, CGC, and other members of the Westat Inherited Bone Marrow Failure Syndromes team for their extensive assistance. We are grateful to the patients and their families for their valuable contributions.

\section{STATEMENT OF FINANCIAL SUPPORT}

This research was supported in part by the Intramural Research Programs of the National Cancer Institute and the Eunice Kennedy Shriver National 


\section{Articles | shankar et al.}

Institute of Child Health and Human Development, of the National Institutes of Health, and by contract HHSN261201100018C with Westat.

Disclosure: The authors declare no conflict of interest.

\section{REFERENCES}

1. Shimamura A, Alter BP. Pathophysiology and management of inherited bone marrow failure syndromes. Blood Rev 2010;24:101-22.

2. Canalis E, Mazziotti G, Giustina A, Bilezikian JP. Glucocorticoid-induced osteoporosis: pathophysiology and therapy. Osteoporos Int 2007;18: 1319-28.

3. Kim MK, Lee JW, Baek KH, et al. Endocrinopathies in transfusionassociated iron overload. Clin Endocrinol 2013;78:271-7.

4. Giri N, Batista DL, Alter BP, Stratakis CA. Endocrine abnormalities in patients with fanconi anemia. J Clin Endocrinol Metab 2007;92:2624-31.

5. McClune BL, Majhail NS. Osteoporosis after stem cell transplantation. Curr Osteoporos Rep 2013;11:305-10.

6. Shepherd JA, Baim S, Bilezikian JP, Schousboe JT. Executive summary of the 2013 international society for clinical densitometry position development conference on body composition. J Clin Densitom 2013;16:489-95.

7. Crabtree NJ, Arabi A, Bachrach LK, et al. Dual-energy X-ray absorptiometry interpretation and reporting in children and adolescents: the revised 2013 ISCD pediatric official positions. J Clin Densitom 2014;17:225-42.

8. Carter DR, Bouxsein ML, Marcus R. New approaches for interpreting projected bone densitometry data. J Bone Miner Res 1992;7:137-45.

9. Melton LJ 3rd, Atkinson EJ, O'Connor MK, O’Fallon WM, Riggs BL. Bone density and fracture risk in men. J Bone Miner Res 1998;13:1915-23.

10. Rose SR, Rutter MM, Mueller R, et al. Bone mineral density is normal in children with fanconi anemia. Pediatr Blood Cancer 2011;57:1034-8.
11. Toiviainen-Salo S, Mayranpaa MK, Durie PR, et al. Shwachman-diamond syndrome is associated with low-turnover osteoporosis. Bone 2007;41: 965-72.

12. Dokal I. Dyskeratosis congenita in all its forms. Br J Haematol 2000;110: 768-79.

13. Alter BP, Giri N, Savage SA, et al. Malignancies and survival patterns in the National Cancer Institute inherited bone marrow failure syndromes cohort study. Br J Haematol 2010;150:179-88.

14. Kuczmarski RJ, Ogden CL, Guo SS, et al. 2000 CDC growth charts for the United States: methods and development. National Center for Health Statistics. Vital Health Stat 11 2002;246:1-190.

15. Zemel BS, Kalkwarf HJ, Gilsanz V, et al. Revised reference curves for bone mineral content and areal bone mineral density according to age and sex for black and non-black children: results of the bone mineral density in childhood study. J Clin Endocrinol Metab 2011;96:3160-9.

16. Sklavos M, Giri N, Stratton P, Alter BP, Pinto LA. Anti-Müllerian hormone deficiency in females with fanconi anemia. Clin Endocrinol Metab 2014;99:1608-14.

17. Zemel BS, Stallings VA, Leonard MB, et al. Revised pediatric reference data for the lateral distal femur measured by the hologic discovery/delphi dual-energy X-ray absorptiometry. J Clin Densitom 2009;12:207-18.

18. Doyard M, Chappard D, Leroyer P, Roth MP, Loreal O, Guggenbuhl P. Decreased bone formation explains osteoporosis in a genetic mouse model of hemochromatosis. PLoS ONE 2016;11:e0148292.

19. Weinstein RS, Jilka RL, Parfitt AM, Manolagas SC. Inhibition of osteoblastogenesis and promotion of apoptosis of osteoblasts and osteocytes by glucocorticoids. Potential mechanisms of their deleterious effects on bone. J Clin Invest 1998;102:274-82. 